\title{
Automated Procedures for the Alignment and Reconstruction of Multiple Tilt Electron Microscopic Tomography Data
}

\author{
Mark H. Ellisman ${ }^{1}$, Daniela Boassa ${ }^{1}$, Phuong Nguyen ${ }^{1}$, Xiaohua Wan ${ }^{1}$, Albert Lawrence ${ }^{1}$, Jason \\ Lanman $^{2}$, Sebastien Phan ${ }^{1}$ \\ ${ }^{1}$ National Center for Microscopy and Imaging Research, University of California San Diego \\ ${ }^{2}$ Department of Biological Sciences, Purdue University
}

Transmission electron microscopy, combined with tomographic techniques, has been used for decades to generate 3D representations of biological structures, with resolutions down to the nanometer range $[1,2]$. Here, we describe a method, extending the Transform-Based Reconstruction (TxBR) method [3,4] whereby large field electron tomography (ET) can be optimized both in terms of data acquisition and processing to deliver higher resolution reconstructions of biological structures in plastic embedded biological specimens, to length scales approaching single proteins.

The quality of an electron tomogram is controlled by different factors including: (i) the interaction between the sample and the electron beam, and (ii) the data acquisition scheme, and its reconstruction process. While there are means to control and limit the effects of multiple scattering events, a more practical solution is to optimize parameters for the best image quality at the higher tilts, and handle noise consequences at lower tilts with additional signal averaging. Traditionally, the ET data acquisition scheme consists of a single or double tilt series, with rather large angle increments ( 2 degrees) between projections in order to minimize specimen exposure to the electron beam. More refined sampling schemes have been developed within the context of Fourier slice theorem to optimize the resolution of the obtained reconstructions [5,6]. Nevertheless, reconstruction artifacts, which result both from the "missing wedge/pyramid" issue and a discretization problem, can only be attenuated through more sampling or eventually by computationally intensive iterative reconstruction methods.

We have developed an n-fold multiple tilt series scheme, generalizing the popular double tilt series, which offers significant improvements over regular single tilt series [7,8], offering both contrast enhancement and artifact reduction. In combination, we have developed automated procedures for the alignment and reconstruction of these multi-tilt series datasets without the need of manual input. We have subsequently applied this method for plastic embedded biological specimens, utilizing the giant amoeba-infecting DNA virus, Mimivirus, as a test system for validating our results.

Since electron-beam damage in plastic-embedded material mostly depends on the dose rate, not the accumulated dose [9], the protocol we have developed is as follows: Take as many micrographs as possible at a low-dose rate over multiple specimen orientations (this could be done at relatively low magnification). An orthogonal rotation (as used in double-tilt series) is the most efficient in eliminating reconstruction artifacts. The exposure time to record a micrograph can be short to prevent any sample drift, and the sample thickness thin enough so the blur caused by multiple scattering events remains minimal for specimen tilts lower than $60^{\circ}$. Then tolerated noise in the raw data is averaged down while the contribution of many micrographs is summed to generate the final reconstruction.

We have further combined this averaging technique with iterative reconstruction to further improve resolution. In particular, we have implemented a SIRT-type iterative method with an adaptive-weighting 
scheme along trajectory paths. Compared to regular filtered back-projection, it requires several iterations of projection/back-projection sequences but contrast enhancement and additional artifact reduction are possible. This leads to the trade-off of whether to acquire a small number of tilt series (typically ranging from 2 to 4) with the iterative approach, or to acquire a large number of tilt series and restrict the reconstruction process to filtered back-projection.

\section{References:}

[1] J. Frank, Electron Tomography. New York: Springer, (2006).

[2] B.F. McEwen et al., "Principles and practice in electron tomography." (2008).

[3] A. Lawrence et al., Journal of structural biology 154.2 (2006): 144-167.

[4] S. Phan et al., Journal of structural biology 180.1 (2012): 154-164.

[5] W.O. Saxton et al., Ultramicroscopy 13.1 (1984), 57-70.

[6] P.A. Penczek and J. Frank, Electron Tomography. Springer New York (2006), 307-330.

[7] D. Mastronarde, Microscopy and Microanalysis 12.S02 (2006): 178-179.

[8] F. Cantele et al., Journal of Structural Biology 169.2 (2010): 192-199.

[9] P.K. Luther, Electron Tomography. Springer New York, 2006. 17-48.

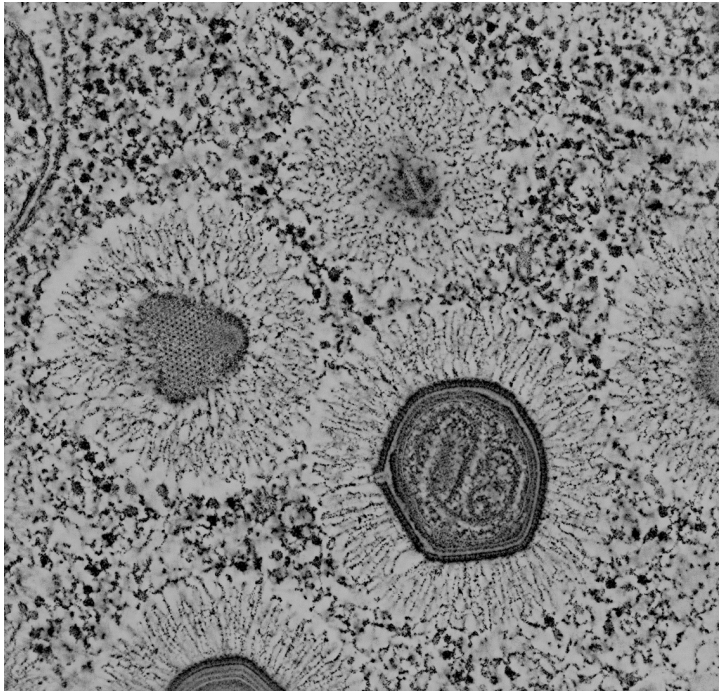

Figure 1. Single slice image from Multiple Tilt Reconstruction of Giant Mimivirus Sample from high pressure frozen, freeze-substituted and epoxy embedded section of an infected cell. Data set was obtained with 0.5 degree increments and reconstructed using TxBR, and automated alignment procedures as well Iterative (weighted SIRT) Methods. Numerous virus particles are seen embedded in a ribosome-rich environment. 